\title{
A system-oriented strategy to enhance electron production of Synechocystis sp. PCC6803 in bio-photovoltaic devices: experimental and modeling insights
}

\author{
Hossein Firoozabadi $^{1}$, Mohammad Mahdi Mardanpour $^{2}$, and Ehsan Motamedian ${ }^{1}$ \\ ${ }^{1}$ Tarbiat Modares University \\ ${ }^{2}$ Sharif University of Technology
}

January 4, 2021

\begin{abstract}
Bio-photovoltaic devices (BPVs) harness photosynthetic organisms to produce bioelectricity in an eco-friendly way. However, their low energy efficiency is still a challenge. A comprehension of metabolic constraints can result in finding strategies for efficiency enhancement. This study presents a systemic approach based on metabolic modeling to design a regulatory defined medium, reducing the intracellular constraints in bioelectricity generation of Synechocystis sp. PCC6803 through the cellular metabolism alteration. The approach identified key reactions that played a critical role in improving electricity generation in Synechocystis sp. PCC6803 by comparing multiple optimal solutions of minimal and maximal NADH generation using two criteria. Regulatory compounds, which controlled the enzyme activity of the key reactions, were obtained from the BRENDA database. The selected compounds were subsequently added to the culture media, and their effect on bioelectricity generation was experimentally assessed. The power density curves for different culture media showed the BPV fed by Synechocystis sp. PCC6803 suspension in BG-11 supplemented with $\mathrm{NH} 4 \mathrm{Cl}$ achieved the maximum power density of $148.27 \mathrm{~mW} \mathrm{~m}-2$. This produced power density was more than 40.5-fold of what was obtained for the BPV fed with cyanobacterial suspension in BG11. The effect of the activators on BPV performance was also evaluated by comparing their overpotential, maximum produced power density, and biofilm morphology under different conditions. These findings demonstrated the crucial role of cellular metabolism in improving bioelectricity generation in BPVs.
\end{abstract}

A system-oriented strategy to enhance electron production of Synechocystis sp. PCC6803 in bio-photovoltaic devices: experimental and modeling insights

Hossein Firoozabadi ${ }^{1}$, Mohammad Mahdi Mardanpour ${ }^{2}$, Ehsan Motamedian ${ }^{1,}$ *

${ }^{1}$ Department of Biotechnology, Faculty of Chemical Engineering, Tarbiat Modares University, P.O. Box 14115-143, Tehran, Iran.

${ }^{2}$ Department of chemical and petroleum engineering, Sharif University of Technology, Tehran, Iran

Corresponding author at: Department of Biotechnology, Faculty of Chemical Engineering, Tarbiat Modares University, P.O. Box 14115-143, Tehran, Iran.

E-mail address: motamedian@modares.ac.ir (E. Motamedian)

\section{Abstract}

Bio-photovoltaic devices (BPVs) harness photosynthetic organisms to produce bioelectricity in an ecofriendly way. However, their low energy efficiency is still a challenge. A comprehension of metabolic constraints can result in finding strategies for efficiency enhancement. This study presents a systemic approach 
based on metabolic modeling to design a regulatory defined medium, reducing the intracellular constraints in bioelectricity generation of Synechocystis sp. PCC6803 through the cellular metabolism alteration. The approach identified key reactions that played a critical role in improving electricity generation in Synechocystis sp. PCC6803 by comparing multiple optimal solutions of minimal and maximal NADH generation using two criteria. Regulatory compounds, which controlled the enzyme activity of the key reactions, were obtained from the BRENDA database. The selected compounds were subsequently added to the culture media, and their effect on bioelectricity generation was experimentally assessed. The power density curves for different culture media showed the BPV fed by Synechocystis sp. PCC6803 suspension in BG-11 supplemented with $\mathrm{NH}_{4} \mathrm{Cl}$ achieved the maximum power density of $148.27 \mathrm{~mW} \mathrm{~m}^{-2}$. This produced power density was more than 40.5-fold of what was obtained for the BPV fed with cyanobacterial suspension in BG-11. The effect of the activators on BPV performance was also evaluated by comparing their overpotential, maximum produced power density, and biofilm morphology under different conditions. These findings demonstrated the crucial role of cellular metabolism in improving bioelectricity generation in BPVs.

Keywords: Regulatory defined medium; Polarization; Cyanobacteria; Genome-scale metabolic modeling; Photosynthetic

\section{Introduction}

Discovering the inherent capability of microorganisms to generate bioelectricity has opened a new era of renewable energy production. Regarding the emergence of microbial fuel cells (MFCs) as a system to extract electrons from the complex organic substrate during the anaerobic biodegradation process, the critical role of particular microorganisms as biocatalysts in wastewater treatment and energy generation has become crystal clear (Slate, Whitehead, Brownson, \& Banks, 2019). Although the MFCs are highly in academic interests (Santoro, Arbizzani, Erable, \& Ieropoulos, 2017), the advantages of bio-photovoltaic devices (BPVs), which can operate without organic sources and carbon dioxide emission, reveal more benefits in comparison with MFCs encountering difficulties of supplying organic feed and carbon dioxide management (Gul \& Ahmad, 2019). In BPVs, the oxygenic photosynthetic organism is being used as a bio-anode catalyst, deriving electrons from water photolysis under the light emission (Qi, Ren, Liang, \& Wang, 2018).

Despite the high potential of BPVs as a promising power generator, the main bottleneck restricting the commercial way is the intense competition of intracellular pathways for energy resources and intrinsic metabolic losses resulting in low power outputs generation (McCormick et al., 2011). Therefore, numerous researches were undertaken to understand the intracellular electron pathways of photosynthetic organisms. Among unicellular photosynthetic organisms, Synechocystis sp. PCC 6803 (henceforth referred to asSynechocystis ), the well-known model organism, received a great deal of attention to investigate the electron transfer pathways (Thiel et al., 2019). In this regard, investigation of inhibitors' effects on the photosynthetic metabolic pathways (Bombelli et al., 2011), creation of mutant strains to redirect electron flux (Bradley, Bombelli, Lea-Smith, \& Howe, 2013), and eliminating photosystem II (Cereda et al., 2014), has been demonstrated in previously published studies to understand electrons generation mechanism in Synechocystis .

Bombelli et al. investigated the use of classic inhibitors such as DCMU, DBMIB, and methyl viologen $(\mathrm{MV})$, to identify the electron transfer chain suggesting that photo-electrons are originated from photolysis of water and end with the reducing form of PS I (Bombelli et al., 2011). Bradley et al. redirected electron flux from competing electron sinks by creating mutant strains of Synechocystis lacking respiratory terminal oxidase complexes and using ferricyanide as an electron acceptor. When terminal oxidase complexes were inactivated, $10 \%$ of the respiratory electron flux was redirected to ferricyanide in the dark condition (Bradley et al., 2013). Cereda et al. studied the effect of photosystem II (PS II) on electron generation through both inhibitor addition and gene deletion. It was also proved that the primary photocurrent generation requires the activation of PS II during water photolysis (Cereda et al., 2014). Moreover, their case study on theSynechocystis revealed the incapability of transferring electrons to the electrode owing to numerous metabolic pathways facing fierce competition with the electrode for the electrons. Thus, extracellular photoelectrons are attributed to a small proportion of the total organism electron flux (Cereda et al., 2014). This matter has been pointed out in Glazier's work that cellular metabolism used only $3 \%$ of electrons produced 
by photosynthetic light reactions (Glazier, 2009), and thus the metabolism has considerable potential for electricity generation. However, switching the cellular metabolism to the generation of NADH is required.

Despite the careful investigations on the photosynthetic electron pathways, the importance of a systemoriented strategy applying metabolic modeling to uncover other metabolic pathways that may contribute to electrons production has been neglected. Mao and Verwoerd applied a metabolic network using flux balance analysis (FBA) to explore the inherent capacity of electron generation for Synechocystis ; however, the lack of experimental works in their studies is evident (Mao \& Verwoerd, 2013).

Enhancing intracellular electron carriers such as NADH and $\mathrm{NAD}^{+}$can be considered a positive approach to enhancing energy generation in bioelectrochemical systems. In this regard, Han et al. regenerated NADH by genetically engineered Clostridium ljungdahlii, and increased the maximum power density of the MFC more than 2-fold (Han, Gao, Ying, \& Zhou, 2016). Moreover, It was demonstrated that the electricity generation ofSynechocystis depended on NADH production, which is profoundly affected by various metabolic pathways (Mao \& Verwoerd, 2013). Therefore, concerning the vital role of metabolic modeling to identify intracellular constraints and switch the cellular metabolism to the generation of $\mathrm{NADH}$, a regulatory defined medium can be developed to shift the cell metabolism to the object of interest (Motamedian, Sarmadi, \& Derakhshan, 2019).

In this study, a system-oriented strategy based on metabolic modeling is used to design a regulatory defined medium, reducing the intracellular constraints in the bioelectricity generation of Synechocystis via the cellular metabolism alteration to improve the NADH generation. A comprehensive investigation is achieved to identify the key reactions that play a critical role in enhancing electricity production inSynechocystis via a linear algorithm for finding multiple optimal solutions (LAMOS) (Mekanik, Motamedian, Fotovat, \& Jafarian, 2019). Consequently, the predicted reactions are regulated by adding enzyme regulators to the culture medium. These compounds regulate the enzyme activity of the target reactions and are found in the BRENDA database (Jeske, Placzek, Schomburg, Chang, \& Schomburg, 2019). Finally, regulators' effect on BPV performance is evaluated by comparing polarization and power density curves under various conditions.

\section{Material and methods}

\section{Metabolic model and in silico simulation}

A genome-scale metabolic network of Synechocystis , named iJN678 (Nogales, Gudmundsson, Knight, Palsson, \& Thiele, 2012), was used to investigate the metabolic pathways. The COBRA Toolbox and the GLPK package were applied to MATLAB 2017b to run the metabolic modeling (Schellenberger et al., 2011). Optimizing the intracellular electrons generation rates was done by defining the NADH production rates as the objective function. The lower bounds of intracellular reversible and irreversible reactions were applied to -1000 and $0 \mathrm{mmol} / \mathrm{gDCW} / \mathrm{h}$, respectively. Moreover, the upper bounds of all intracellular reactions were set to $1000 \mathrm{mmol} / \mathrm{gDCW} / \mathrm{h}$. Photoautotrophic conditions were simulated by constraining the photon uptake rate to $52 \mathrm{mmol} / \mathrm{gDCW} / \mathrm{h}$, and bicarbonate uptake rates were set to $3.7 \mathrm{mmol} / \mathrm{gDCW} / \mathrm{h}$ as the only carbon source. The lower and upper bounds of exchange reactions including $\mathrm{Co}^{2+}, \mathrm{Fe}^{2+}, \mathrm{Fe}^{3+}, \mathrm{H}^{+}, \mathrm{Na}^{+}$, $\mathrm{Ni}^{2+}, \mathrm{Cu}^{2+}, \mathrm{Zn}^{2+}, \mathrm{Ca}^{2+}, \mathrm{Mg}^{2+}, \mathrm{Mn}^{2+}, \mathrm{O}_{2}, \mathrm{H}_{2} \mathrm{O}$, Molybdate, Potassium, Nitrate, Phosphate, and Sulfate remained unconstrained by -1000 and $1000 \mathrm{mmol} / \mathrm{gDCW} / \mathrm{h}$, respectively. Hence the uptake and secretion of the mentioned metabolites were allowed (Nogales et al., 2012).

\section{Identifying effective reactions and designing the regulatory defined medium}

As previously described (Mekanik et al., 2019), LAMOS was employed to calculate multiple optimal flux distributions and determine effective reactions (Motamedian \& Naeimpoor, 2018). For this, the optimal biomass formation rate was calculated to be $0.0884 \mathrm{mmol} / \mathrm{gDCW} / \mathrm{h}$, and the growth rate was initially bound to $90 \%$ of the optimal growth rate; moreover, a threshold of $10^{-8}$ was considered to determine active reactions. Thus, 10,000 optimal solutions were found for two conditions, including the maximum and the minimum rates of $\mathrm{NADH}$ production (i.e., $\mathrm{NADH} \rightarrow \mathrm{NAD}^{+}+H^{+}+2 e^{-}$). Then, for each mentioned condition, the flux variability for each reaction was determined (Fig. 1), and two criteria were applied to identify the 
candidate reactions for up or down regulations.

As demonstrated in Fig.1, a reaction is suitable to be up-regulated if its minimum flux in the maximum case (i.e., maximum NADH production rate) is higher than the maximum flux in the minimum case (i.e., minimum NADH production rate). In contrast, a reaction is proper to be down-regulated if its minimum flux in the minimum case is higher than the maximum flux in the maximum one.

The activity difference for each reaction was regarded as the second criterion and was defined as a fraction of entire optimal solutions wherein the reaction was indicated to be active. Therefore, those reactions were taken into consideration that possessed the absolute value of activity difference equal to 1 . In this regard, the activity difference of 1 for a reaction indicated that the reaction was entirely active in every 10,000 optimal solutions of maximum NADH production while it was completely inactive in all optimal solutions of minimum NADH production. On the other hand, the reaction, which activated and inactivated entirely in every 10,000 optimal solutions of minimum and maximum NADH production, respectively, possessed the activity difference of -1 .

(Fig. 1)

The effect of up (down)-regulation of the predicted reactions was experimentally investigated by adding regulators to the culture medium. These regulatory compounds found in the BRENDA database affected the target enzyme activity controlling the target reaction, which is predicted to result in the overproduction of NADH and, consequently, electricity enhancement. Therefore, the investigation of the regulators' impact on electrons overproduction by Synechocystis is another goal of the study.

\section{Bio-photovoltaic cell (BPV) assembly}

The air-cathode single chamber BPV with a working volume of $8.8 \mathrm{~cm}^{3}$, height of $2.2 \mathrm{~cm}$, and electrodes surface of $4 \mathrm{~cm}^{2}$ was fabricated. The anodic and cathodic chambers were fabricated from polymethyl methacrylate (PMMA). The graphite-coated stainless steel mesh (mesh size 400) was used as the anode and cathode electrodes (Naraghi, Yaghmaei, Mardanpour, \& Hasany, 2015). The cathode was treated by the procedures described in (Cheng, Liu, \& Logan, 2006) to attain $0.5 \mathrm{mg} \mathrm{cm}^{-2} \mathrm{Pt}$ loading. A schematic of the $\mathrm{BPV}$ is shown in Fig. 2.

(Fig. 2)

\section{Microorganism and culture media}

Synechocystis sp. PCC6803 (subsequently referred to asSynechocystis ) was obtained from Ariyan Gostar Research Corporation. All cultivation experiments conducted in the BG-11 medium described in the work of Bombelli et al. (Bombelli et al., 2011). In this regard, liquid seed culture was obtained periodically, which was then used for the inoculation of fresh liquid culture. Subsequently, $2 \mathrm{ml}$ of seed culture was inoculated into $100 \mathrm{ml}$ flasks containing $18 \mathrm{ml}$ of fresh BG-11. All incubations were carried out at $150 \mathrm{rpm}$ and $29{ }^{\circ} \mathrm{C}$, which were continuously kept under the illumination of a red light bulb (630 nm) with 6000 lux lighting. It was demonstrated that red light wavelengths have a significant impact on maintaining photosynthetic reactions (Sarcina, Bouzovitis, \& Mullineaux, 2006).

\section{Inoculation and BPV operation}

In all experiments, after five days of cultivation, cyanobacterial cells were harvested during exponential growth $\left(\mathrm{OD}_{750}=3.5 \pm 0.1\right)$ and centrifuged for $15 \mathrm{~min}$ at $3000 \mathrm{~g}$. Then, regarding the experiment to be studied, Synechocystiscells resuspended in fresh BG-11 or regulatory BG-11. With regard to the regulatory BG-11 medium, the selected regulatory compounds were supplemented to fresh BG-11 according to the concentrations obtained from Brenda to evaluate their effect on electricity generation. Thus, the BPV was inoculated by different culture media types under the batch mode operation and open circuit conditions. The inoculation continued until a stable cell potential peak was obtained, indicating successful microbial enrichment fulfillment and cyanobacterial cells stabilization. The bioelectrochemical experiments were conducted under 6000 lux lighting of red light $(630 \mathrm{~nm})$. High-intensity lighting and red light illumination were reported to 
have a positive effect on increasing power generation of the bioelectrochemical systems (Madiraju, Lyew, Kok, \& Raghavan, 2012).

\section{Results and discussion}

\section{Identifying the effective reactions in NADH production}

The appropriate reactions contributing to an increase in the intracellular NADH generation of Synechocystis were predicted and shown in Fig. 3A. The reactions depicted on the top possessed the activity difference of 1. Furthermore, their minimum flux under maximum NADH production rate is more than their maximum flux under the minimum NADH production rate (Fig. 1A), and thus there were proposed for up-regulation. In contrast, down-regulation should be considered for those reactions revealed on the bottom with the activity difference of -1 in which their minimum flux under minimum NADH production rate is more than the maximum flux under maximum $\mathrm{NADH}$ production rate (Fig. 1B). The maximum and minimum NADH production rates were calculated to be 2.1 and $0 \mathrm{mmol} / \mathrm{gDCW} / \mathrm{h}$, respectively. The predicted reactions were prepared in more detail in the Supplementary file (Tables S1 and S2). Moreover, a complete name of all reactions and metabolites were presented in the Supplementary file (Tables S3 and S4), respectively.

(Fig. 3)

The predicted effective metabolic pathways for enhancing NADH are schematically represented in Fig. 3B. Herein, two crucial metabolic pathways for converting acetate (ac) to acetyl-CoA (accoa) that possessed a significant activity difference were selected. As shown in Fig. 3C, acetate could be consumed via the acetylCoA synthetase (ACS) by converting ATP to AMP. Besides, this metabolite can also be used through a series of reactions, including acetate kinase $\left(\mathrm{ACKr}_{f}\right)$ and phosphotransacetylase (PTAr_b), by conversion of ATP to ADP (Fig. 3C). Thus, acetate kinase and phosphotransacetylase were recommended to produce acetyl-CoA, which then enters the TCA cycle and causes NADH to form via malate dehydrogenase (MDH_f).

The systemic approach also recommended that glycerol 3-phosphate (glyc3p) played a vital role in NADH generation. As demonstrated in Fig. 3B, a red dashed line that starts with glyoxalate carboligase pathway (GLXCL) and ends with glycerol kinase (GLYK), consumed three moles NADH along with one mole of ATP to produce glycerol 3-phosphate. These pathways occur as a series of reactions that each reaction possesses the same flux through the metabolic network leading to $0 \mathrm{mmol} / \mathrm{gDCW} / \mathrm{h} \mathrm{NADH}$ generation (Table 1). Therefore, they are regarded as proper candidates to be down-regulated. Moreover, glycerol 3phosphate could also be produced by glycerol-3-phosphate dehydrogenase (G3PD2_b), which consumes only one mole of NADH without ATP consumption. Green arrows show this pathway in Fig. 3B. Glycerol-3phosphate dehydrogenase possesses the same reaction rates as the mentioned series reactions but leads to $2.1 \mathrm{mmol} / \mathrm{gDCW} / \mathrm{h}$ NADH generation (Table 1). Thus, its up-regulation should be taken to prevent NADH and ATP loss via other metabolic pathways.

\section{(Table 1)}

A further investigation into metabolic pathways depicted in (Fig. 3B), showed that the generated glycerol 3-phosphate produces acyl carrier protein (acp), which is then used to form CoA, which can be utilized by (PTAr_b). Furthermore, the hypothetical glycerol-3-phosphate acyltransferase reactions (G3PAT Rxns) comprise nine reactions generating acyl carrier protein, in which their details were mentioned in the Supplementary file (Table S5).

\section{Microbial enrichment and open circuit potential (OCP) monitoring}

With regard to the introduced reactions by metabolic modeling in Fig. 3A, phosphotransacetylase and glutamate dehydrogenase were taken into consideration because of the availability of their regulators. As reported in Brenda and previously published studies, $40 \mathrm{mM} \mathrm{NH}_{4} \mathrm{Cl}$ resulted in 3-fold stimulation of phosphotransacetylase (Brinsmade \& Escalante-Semerena, 2007), and 50mM KCl led to an increase in $170 \%$ activity of glutamate dehydrogenase (Bhuiya et al., 2000). Hence, three suspensions for microbial enrichment were used including Synechocystis + BG-11, Synechocystis + BG-11 + 50mM KCl, and Synechocystis 
+ BG-11 $+40 \mathrm{mM} \mathrm{NH}_{4} \mathrm{Cl}$. Having concluded an effective biofilm formation during open circuit conditions (Zhang, Zhu, Li, Liao, \& Ye, 2011), which established a uniform biofilm facilitating substrate diffusion and electron transfer, the microbial enrichment of the BPV has been done by monitoring the open circuit potential (OCP). Thus, the OCP evolution of the three culture media was shown in Fig. 4.

(Fig. 4)

Considering the OCP of the sole culture medium of BG-11, the addition of activators (i.e., $\mathrm{KCl}$ or $\mathrm{NH}_{4} \mathrm{Cl}$ ) bring about a significant increase in the OCP of the BPV. Since Nernst's equation depicting the effective parameters on the electrochemical OCP, the higher cell potentials strongly depend on the activation of redox species implying the critical role of $\mathrm{KCl}$ and $\mathrm{NH}_{4} \mathrm{Cl}$ in this increment.

By observing a noticeable decrease in OCP, the fresh medium was injected into the BPV, as shown by arrows in Fig. 4. The replacement of the fresh culture medium with the old one causes an increase in the OCP trend. Since the BPV operated in the batch mode with high cell density $\left(\mathrm{OD}_{750}=3.5 \pm 0.1\right)$, the potential reduction can be attributed to the depletion of nutrients; thus, fresh medium injection compensates for the potential drop. This phenomenon was also shown previously in the work of Madiraju et al. (Madiraju et al., 2012), in which replenishing minerals led to an increase in power density. Moreover, for the culture media with activator, this abrupt growth in the OCP was higher than the sole culture medium of BG-11. A comparison between Figs. 4A and $4 \mathrm{~B}$ reveal that the culture medium of Synechocystis + BG-11 $+40 \mathrm{mM}$ $\mathrm{NH}_{4} \mathrm{Cl}$ obtained the higher OCP and more prolonged stationary phase. This issue puts the emphasis on the more impact of this culture medium on the cell potential compared to the BG- $11+50 \mathrm{mM} \mathrm{KCl}$.

\section{Polarization and power density curves}

The effect of external resistance on the BPV performance fed with various culture media types was investigated by monitoring current evolution. Fig. 5A depicted the current evolution of Synechocystis for BG-11, BG- $11+\mathrm{KCl}$, and $\mathrm{BG}-11+\mathrm{NH}_{4} \mathrm{Cl}$ culture media at 500, 500, and $750 \mathrm{k} \Omega$ external resistances, respectively. The presence of $\mathrm{KCl}$ increased the maximum produced current of the BPV by more than $8.8 \%$ compared with its value when the initial culture medium of BG-11 was fed. The addition of $\mathrm{NH}_{4} \mathrm{Cl}$ to BG-11 had a remarkable influence on the current production and increased the maximum current density of the cell more than 2-fold compared with the BPV fed by BG-11. This incremental phenomenon was obtained at a higher external resistance of $750 \mathrm{k} \Omega$, which was comparable to the other media implying more increase have to be obtained in the same external resistance (i.e., $500 \mathrm{k} \Omega$ ).

Additionally, the stationary phase in the current evolution (shown by two dashed horizontal arrows in Fig. $5 \mathrm{~A}$ ) of the BG-11+ $\mathrm{NH}_{4} \mathrm{Cl}$ fed BPV was longer compared to the BPV cultured BG-11 and BG-11+KCl, indicating a stable current density by extension feed replacement.

(Fig. 5)

The BPV overpotentials (including activation, ohmic, and concentration) can be investigated by applying different external resistances to polarize the cell. The polarization curves of four types of BPV feed was illustrated in Fig. 5B. The abrupt reductions of cell potentials in the polarization curves of BG-11+Synechocystis and $\mathrm{BG}-11+\mathrm{KCl}+$ Synechocystis indicate the high activation overpotential to extract the electron from the cellular metabolism. This type of overpotential was remarkably higher than two other overpotentials (i.e., ohmic and concentration overpotentials) characterized at the middle and end of polarization curves. Activation overpotential represents the energy that microbes required to transfer electrons from their surface to the electrode (Logan et al., 2006). These high drops corroborate the hypothesis presented in previous researches (Cereda et al., 2014; McCormick et al., 2011), indicating the incapability of Synechocystis to transfer electrons from its membrane due to the competition of numerous metabolic pathways for energy resources. Moreover, the absence and presence of cyanobacteria in the BG-11 culture medium demonstrated the incapability ofSynechocystis in generating electrons. Although the addition of $\mathrm{KCl}$ increased the current density, the initial sharp reduction could not be compensated. However, for the BPV fed BG- $11+\mathrm{NH}_{4} \mathrm{Cl}+$ Synechocystis 
, the cell potential reduction was exceedingly made up, revealing the impressive role of $\mathrm{NH}_{4} \mathrm{Cl}$ to compensate for the activation loss of metabolic reactions and extract electrons from light and water.

Furthermore, the higher current densities obtained at lower external resistances show the augmented electron production and accentuate the stimulation of cell electrogenesis metabolic pathways. Low activation losses owned to the fact that phosphotransacetylase had a crucial role in converting acetate to acetyl-CoA, assisting the cell to maintain a balance between biosynthesis and energy production (El-Mansi, Cozzone, Shiloach, \& Eikmanns, 2006). Therefore, the overexpression of this enzyme by $\mathrm{NH}_{4} \mathrm{Cl}$ could facilitate electrons transfer.

Moreover, the occurrence of the overshoot phenomenon resulting from sudden electron depletion at low external resistances that cannot be compensated by microorganisms (Hong, Call, Werner, \& Logan, 2011) was observed in the BPV fed with all four types of culture media (Fig. 5B). The overshoot in the BPV fed with BG-11+KCl+Synechocystis led to a more than $31 \%$ decrease in the current density (from 0.1913 to 0.1306 $\mathrm{mA} \mathrm{m}{ }^{-2}$ ). The culture medium used $\mathrm{NH}_{4} \mathrm{Cl}$ as an activator showed a better performance in compensating electron depletions and only brought about a $6.6 \%$ decrease in the produced current. This is another evidence emphasizing the crucial role of $\mathrm{NH}_{4} \mathrm{Cl}$, which improves Synechocystiselectrogenesis metabolic pathways.

The power density curves for three culture media were shown in Fig. 5C to Fig. 5E. The BPV fed by BG-11+ $\mathrm{NH}_{4} \mathrm{Cl}+$ Synechocystis achieved the maximum power density of $148.27 \mathrm{~mW} \mathrm{~m}^{-2}$, which is more than 40.5-fold of what was obtained for the BPV fed with BG-11+Synechocystis (Fig. 5C).

The maximum produced power density for BG-11+KCl+Synechocystiswas $10.97 \mathrm{~mW} \mathrm{~m}^{-2}$ (Fig. 5D). Despite the higher salt concentration, this power density was 13.5 times lower than the BPV fed by BG$11+\mathrm{NH}_{4} \mathrm{Cl}+$ Synechocystis . Achieving a higher power density by $\mathrm{NH}_{4} \mathrm{Cl}$ compared to $\mathrm{KCl}$ can be related to the fact that $\mathrm{KCl}$ had an adverse effect on some critical metabolic pathways, which is discussed in the Supplementary file (Table S6). Therefore, with regard to the role of $\mathrm{KCl}$ in the stimulation of glutamate dehydrogenase, it cannot be helpful in electrogenesis metabolism as much as $\mathrm{NH}_{4} \mathrm{Cl}$.

Furthermore, to scrutinize the effect of $\mathrm{NH}_{4} \mathrm{Cl}$ on diminishing intracellular constraints, Synechocystis was removed from the BPV. In this case, the maximum produced power density for BG- $11+\mathrm{NH}_{4} \mathrm{Cl}$ was 13.72 $\mathrm{mW} \mathrm{m}^{-2}$ (Fig. 5E). This power density was more than 10-fold lower than the maximum power density of the BPV fed with BG-11+NH $\mathrm{NH}_{4} \mathrm{Cl}+$ Synechocystis. The abiotically produced power density is mainly attributed to the medium salinity (Logan, 2009; Logan et al., 2006). Therefore, the presence of $\mathrm{NH}_{4} \mathrm{Cl}$ results in the metabolic stimulation of Synechocystis rather than the anolyte conductivity improvement. Thus, the addition of $\mathrm{NH}_{4} \mathrm{Cl}$ can play a critical role in shifting the Synechocystis metabolism to produce more electricity.

\section{Biofilm Morphology}

FESEM micrographs of Synechocystis in BG-11 and BG-11+ $\mathrm{NH}_{4} \mathrm{Cl}$ were illustrated in Fig. 6. In both cases, a small portion of cells attached to the anode's surface. This low biofilm formation reported here is similar to those investigated by (McCormick et al., 2011) in which $8.5 \%$ of Synechocystis attached to the anode's surface made of indium tin oxide-coated polyethylene terephthalate (ITO-PET). Consequently, the addition of $\mathrm{NH}_{4} \mathrm{Cl}$ to BG-11 had a small impact on the biofilm morphology, and the observed power density enhancement is mainly attributed to the improvement of electrogenesis metabolic pathways.

(Fig. 6)

\section{Conclusion}

The system-oriented strategy based on metabolic modeling discloses the effective reactions and enzyme activators to enhance the bioelectricity production in BPVs. These activators could provoke theSynechocystis as a strain engaged in noticeable intrinsic metabolic losses to reduce its activation overpotential significantly and accelerate bioelectricity generation.

The overexpression of the phosphotransacetylase by $\mathrm{NH}_{4} \mathrm{Cl}$ exceedingly facilitates electrons transfer and stimulate cell electrogenesis metabolic pathways, which brings about lower activation losses. Although $\mathrm{KCl}$ led to an increase in glutamate dehydrogenase activity, the experimental results showed that it could not 
be helpful in electrogenesis metabolism as much as $\mathrm{NH}_{4} \mathrm{Cl}$. Additionally, the investigation of overshoot occurrence in the BPV demonstrated the culture medium used $\mathrm{NH}_{4} \mathrm{Cl}$ as an activator produced a better performance in compensating electron depletions. Furthermore, the addition of $\mathrm{NH}_{4} \mathrm{Cl}$ had a small effect on the biofilm morphology.

Consequently, unlike the addition of classic inhibitors such as DCMU and DBMIB, which were used to investigate the source of electrons in the photosynthetic organism, the supplementation of regulators identified by the systemic approach had a significant impact on improving electrical power generation. It is worth to mention that the produced power density of Synechocystis is still relatively lower than exoelectrogenic bacteria such as Shewanella or Geobacter. However, this method can be implemented in enhancing exoelectrogenic bacteria bioelectricity generation.

\section{Availability of data and materials}

All data generated or analysed during this study are included in this published article.

\section{Competing interests}

The authors declare that they have no competing interests.

\section{Author contributions}

H.F., M.M.M., \& E.M. designed and performed research and wrote the paper.

\section{References}

Bhuiya, M. W., Sakuraba, H., Kujo, C., Nunoura-Kominato, N., Kawarabayasi, Y., Kikuchi, H., \& Ohshima, T. (2000). Glutamate dehydrogenase from the aerobic hyperthermophilic archaeon Aeropyrum pernix K1: enzymatic characterization, identification of the encoding gene, and phylogenetic implications. $E x-$ tremophiles, 4 (6), 333-341.

Bombelli, P., Bradley, R. W., Scott, A. M., Philips, A. J., McCormick, A. J., Cruz, S. M., . . Cameron, P. J. (2011). Quantitative analysis of the factors limiting solar power transduction by Synechocystis sp. PCC 6803 in biological photovoltaic devices. Energy 83 Environmental Science, 4 (11), 4690-4698.

Bradley, R. W., Bombelli, P., Lea-Smith, D. J., \& Howe, C. J. (2013). Terminal oxidase mutants of the cyanobacterium Synechocystis sp. PCC 6803 show increased electrogenic activity in biological photo-voltaic systems. Physical Chemistry Chemical Physics, 15 (32), 13611-13618.

Brinsmade, S. R., \& Escalante-Semerena, J. C. (2007). In vivo and in vitro analyses of single-amino acid variants of the Salmonella enterica phosphotransacetylase enzyme provide insights into the function of its N-terminal domain. Journal of Biological Chemistry, 282 (17), 12629-12640.

Cereda, A., Hitchcock, A., Symes, M. D., Cronin, L., Bibby, T. S., \& Jones, A. K. (2014). A bioelectrochemical approach to characterize extracellular electron transfer by Synechocystis sp. PCC6803. PLoS One, 9 (3).

Cheng, S., Liu, H., \& Logan, B. E. (2006). Increased performance of single-chamber microbial fuel cells using an improved cathode structure.Electrochemistry communications, 8 (3), 489-494.

El-Mansi, M., Cozzone, A. J., Shiloach, J., \& Eikmanns, B. J. (2006). Control of carbon flux through enzymes of central and intermediary metabolism during growth of Escherichia coli on acetate. Current opinion in microbiology, 9 (2), 173-179.

Glazier, D. S. (2009). Metabolic level and size scaling of rates of respiration and growth in unicellular organisms. Functional Ecology, 23 (5), 963-968.

Gul, M. M., \& Ahmad, K. S. (2019). Bioelectrochemical systems: Sustainable bio-energy powerhouses. Biosensors and Bioelectronics, 111576. 
Han, S., Gao, X.-y., Ying, H.-j., \& Zhou, C. C. (2016). NADH gene manipulation for advancing bioelectricity in Clostridium ljungdahlii microbial fuel cells. Green Chemistry, 18 (8), 2473-2478.

Hong, Y., Call, D. F., Werner, C. M., \& Logan, B. E. (2011). Adaptation to high current using low external resistances eliminates power overshoot in microbial fuel cells. Biosensors and Bioelectronics, 28 (1), 71-76.

Jeske, L., Placzek, S., Schomburg, I., Chang, A., \& Schomburg, D. (2019). BRENDA in 2019: a European ELIXIR core data resource. Nucleic acids research, 47 (D1), D542-D549.

Logan, B. E. (2009). Exoelectrogenic bacteria that power microbial fuel cells. Nature Reviews Microbiology, 7 (5), 375-381.

Logan, B. E., Hamelers, B., Rozendal, R., Schröder, U., Keller, J., Freguia, S., . . . Rabaey, K. (2006). Microbial fuel cells: methodology and technology. Environmental science E technology, 40 (17), 5181-5192.

Madiraju, K. S., Lyew, D., Kok, R., \& Raghavan, V. (2012). Carbon neutral electricity production by Synechocystis sp. PCC6803 in a microbial fuel cell. Bioresource technology, 110 , 214-218.

Mao, L., \& Verwoerd, W. S. (2013). Genome-scale stoichiometry analysis to elucidate the innate capability of the cyanobacterium Synechocystis for electricity generation. Journal of industrial microbiology $\mathcal{E}^{3}$ biotechnology, 40 (10), 1161-1180.

McCormick, A. J., Bombelli, P., Scott, A. M., Philips, A. J., Smith, A. G., Fisher, A. C., \& Howe, C. J. (2011). Photosynthetic biofilms in pure culture harness solar energy in a mediatorless bio-photovoltaic cell (BPV) system. Energy \& Environmental Science, 4 (11), 4699-4709.

Mekanik, M., Motamedian, E., Fotovat, R., \& Jafarian, V. (2019). Reconstruction of a genome-scale metabolic model for Auxenochlorella protothecoides to study hydrogen production under anaerobiosis using multiple optimal solutions. International Journal of Hydrogen Energy, 44 (5), 2580-2591.

Motamedian, E., \& Naeimpoor, F. (2018). LAMOS: A linear algorithm to identify the origin of multiple optimal flux distributions in metabolic networks. Computers $\& 3$ Chemical Engineering, 117 , 372-377.

Motamedian, E., Sarmadi, M., \& Derakhshan, E. (2019). Development of a regulatory defined medium using a system-oriented strategy to reduce the intracellular constraints. Process Biochemistry, 87, 10-16.

Naraghi, Z. G., Yaghmaei, S., Mardanpour, M. M., \& Hasany, M. (2015). Produced water treatment with simultaneous bioenergy production using novel bioelectrochemical systems. Electrochimica Acta, 180 , 535544 .

Nogales, J., Gudmundsson, S., Knight, E. M., Palsson, B. O., \& Thiele, I. (2012). Detailing the optimality of photosynthesis in cyanobacteria through systems biology analysis. Proceedings of the National Academy of Sciences, 109 (7), 2678-2683.

Qi, X., Ren, Y., Liang, P., \& Wang, X. (2018). New insights in photosynthetic microbial fuel cell using anoxygenic phototrophic bacteria. Bioresource technology, 258, 310-317.

Santoro, C., Arbizzani, C., Erable, B., \& Ieropoulos, I. (2017). Microbial fuel cells: from fundamentals to applications. A review.Journal of power sources, 356 , 225-244.

Sarcina, M., Bouzovitis, N., \& Mullineaux, C. W. (2006). Mobilization of photosystem II induced by intense red light in the cyanobacterium Synechococcus sp PCC7942. The Plant Cell, 18 (2), 457-464.

Schellenberger, J., Que, R., Fleming, R. M., Thiele, I., Orth, J. D., Feist, A. M., . . Rahmanian, S. (2011). Quantitative prediction of cellular metabolism with constraint-based models: the COBRA Toolbox v2. 0. Nature protocols, 6 (9), 1290.

Slate, A. J., Whitehead, K. A., Brownson, D. A., \& Banks, C. E. (2019). Microbial fuel cells: An overview of current technology. Renewable and sustainable energy reviews, 101, 60-81. 
Thiel, K., Patrikainen, P., Nagy, C., Fitzpatrick, D., Pope, N., Aro, E.-M., \& Kallio, P. (2019). Redirecting photosynthetic electron flux in the cyanobacterium Synechocystis sp. PCC 6803 by the deletion of flavodiiron protein Flv3. Microbial cell factories, 18 (1), 189.

Zhang, L., Zhu, X., Li, J., Liao, Q., \& Ye, D. (2011). Biofilm formation and electricity generation of a microbial fuel cell started up under different external resistances. Journal of power sources, 196 (15), 6029-6035.

Table 1. Comparison of reaction rates for generating glycerol 3-phosphate. The fluxes are presented in the unit of $\mathrm{mmol} / \mathrm{gDCW} / \mathrm{h}$.

\begin{tabular}{|c|c|c|c|c|}
\hline Reaction Name & $\begin{array}{l}\text { Reaction } \\
\text { Description }\end{array}$ & $\begin{array}{l}\text { Reaction of } \\
\text { G3PD2_b and the } \\
\text { objective function } \\
\text { are maximal } \\
(\mathrm{mmol} / \mathrm{gDCW} / \mathrm{h}) \text {. }\end{array}$ & $\begin{array}{l}\text { Reaction of } \\
\text { G3PD2_b and the } \\
\text { objective function } \\
\text { are zero } \\
(\mathrm{mmol} / \mathrm{gDCW} / \mathrm{h}) \text {. }\end{array}$ & Activity Difference \\
\hline$\overline{\text { GLXCL }}$ & $\begin{array}{l}\text { Glyoxalate } \\
\text { carboligase }\end{array}$ & 0 & 0.0138 & -0.9618 \\
\hline TRSARr_f & $\begin{array}{l}\text { Tartronate } \\
\text { semialdehyde } \\
\text { reductase }\end{array}$ & 0 & 0.0138 & -0.9618 \\
\hline GLYALDDr_b & $\begin{array}{l}\text { D-glyceraldehyde } \\
\text { dehydrogenase }\end{array}$ & 0 & 0.0138 & -1 \\
\hline ALCD19_f & $\begin{array}{l}\text { Alcohol } \\
\text { dehydrogenase }\end{array}$ & 0 & 0.0138 & -1 \\
\hline GLYK & Glycerol kinase & 0 & 0.0138 & -1 \\
\hline G3PD2_b & $\begin{array}{l}\text { Glycerol-3- } \\
\text { phosphate } \\
\text { dehydrogenase }\end{array}$ & 0.0138 & 0 & 1 \\
\hline NADH_pro & & 2.1 & 0 & 1 \\
\hline
\end{tabular}

The suffixes $f$ and _b indicate the forward and backward directions of a reaction, respectively.

Figure Captions 
(A)

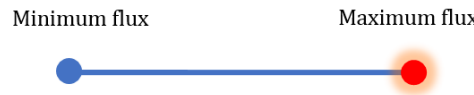

Objective function: Minimization of NADH production rate
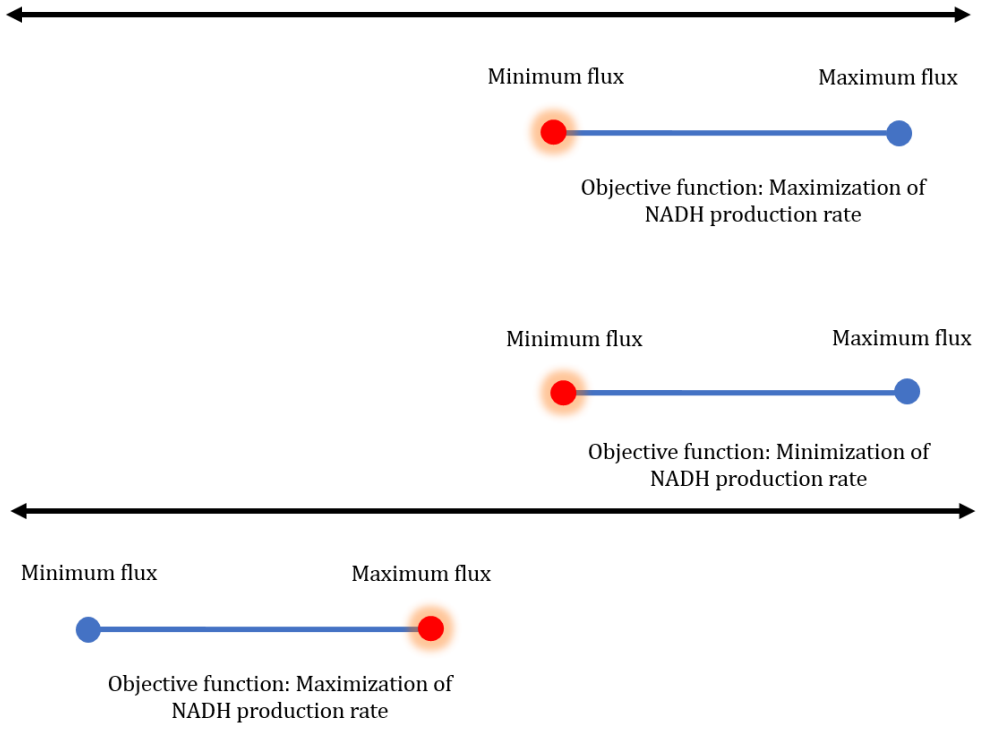

Fig. 1. Comparison of flux variability for each reaction at the minimum and maximum rates of NADH production reaction to finding appropriate reactions for (A) up-regulation, and (B) down-regulation. Compared rates were highlighted in red.

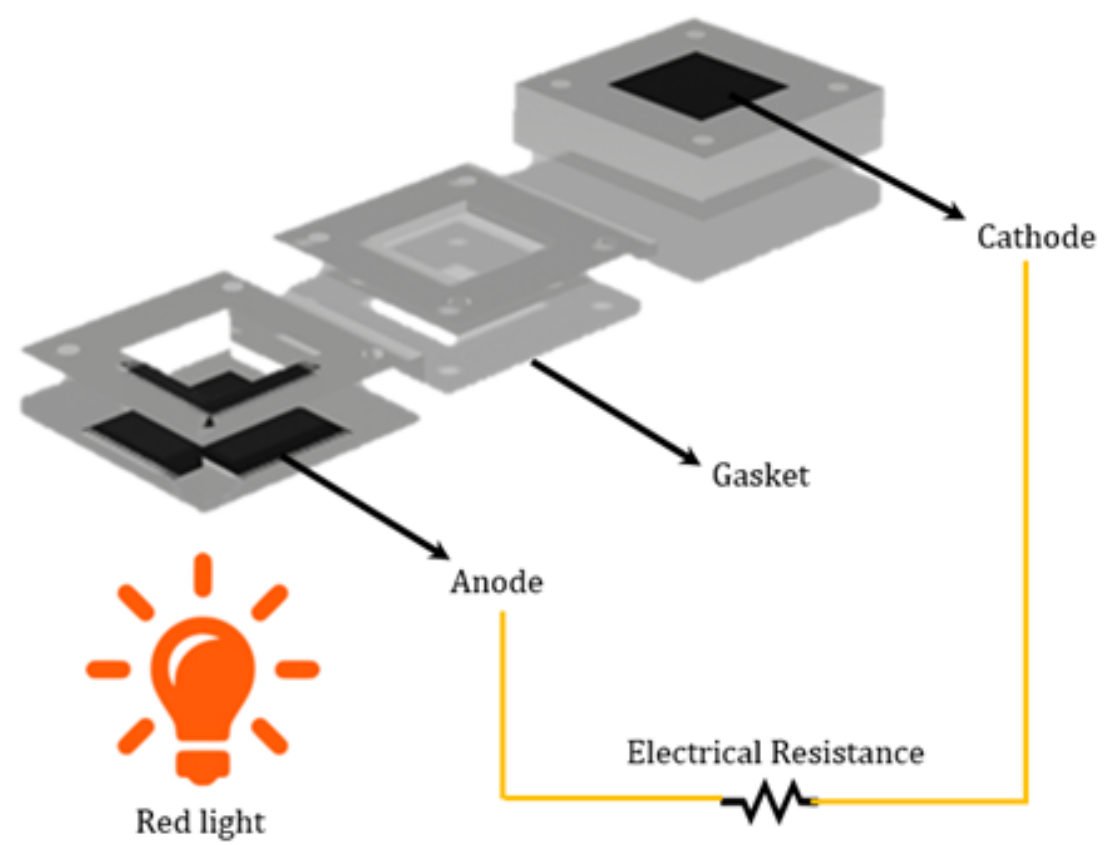


Fig. 2. The schematic of the bio-photovoltaic device (BPV).

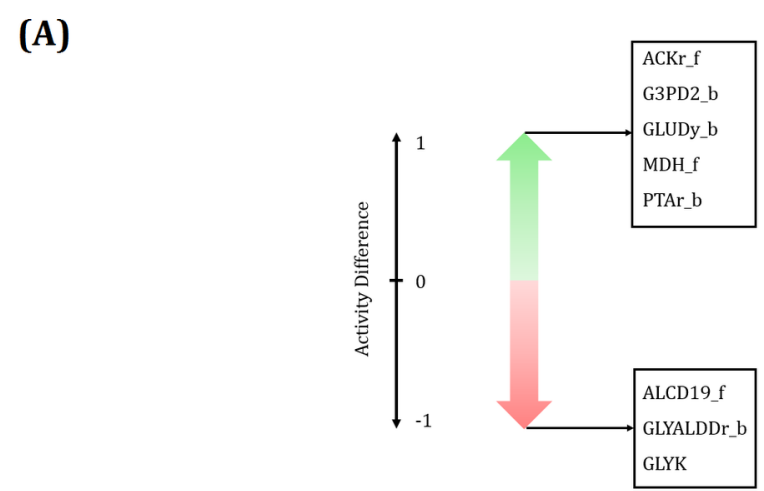

(B)

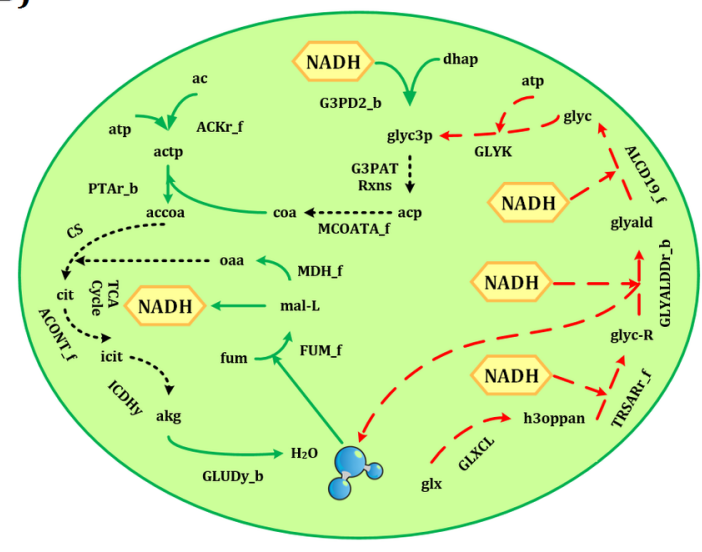

(C)

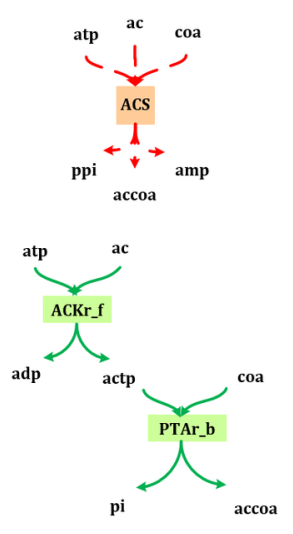

Fig. 3. (A) Appropriate reactions for up-regulation (top) and down-regulation (bottom) predicted by the proposed systemic approach under phototrophic condition. (B) An overview of the metabolic pathways influencing NADH production; the reactions indicated by green arrows are suitable candidates for up-regulation, whereas red dashed lines should be down-regulated. Besides, reactions that do not contribute to NADH enhancement were shown with the black dotted line. (C) Acetate consumption via ACS, and series reactions of ACKr_f and PTAr_b. The suffixes _f and _b were used for reactions that occurred in the forward and backward directions, respectively. The entire name of all reactions and metabolites was given in the Supplementary file (Tables S3 and S4). 
(A)

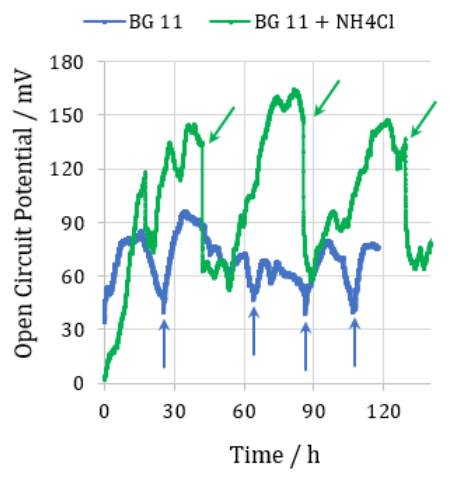

(B)

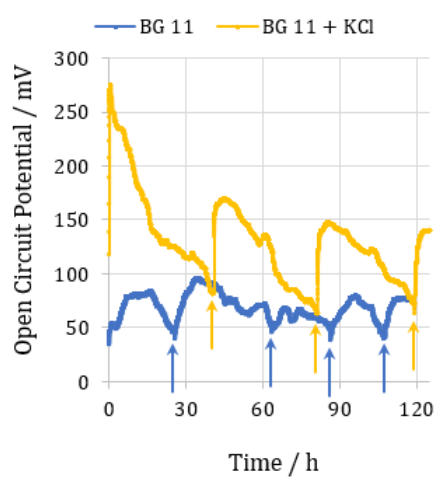

Fig. 4. The open circuit potential (OCP) evolution of three used suspensions for microbial enrichment. (A) Synechocystis + BG-11 and Synechocystis + BG-11 + 40mM NH ${ }_{4} \mathrm{Cl}$, (B) Synechocystis + BG-11 and Synechocystis + BG-11 + 50mM KCl.

(A)

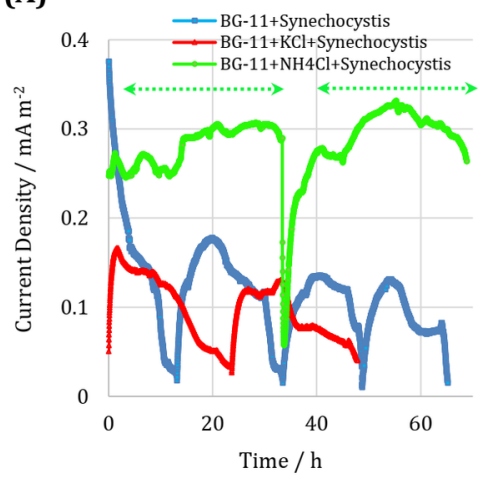

(C)
(B)

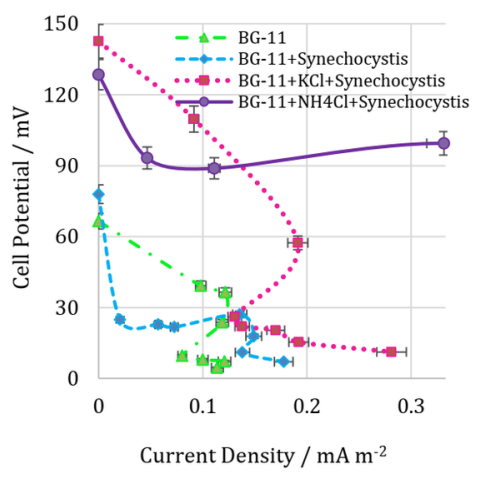

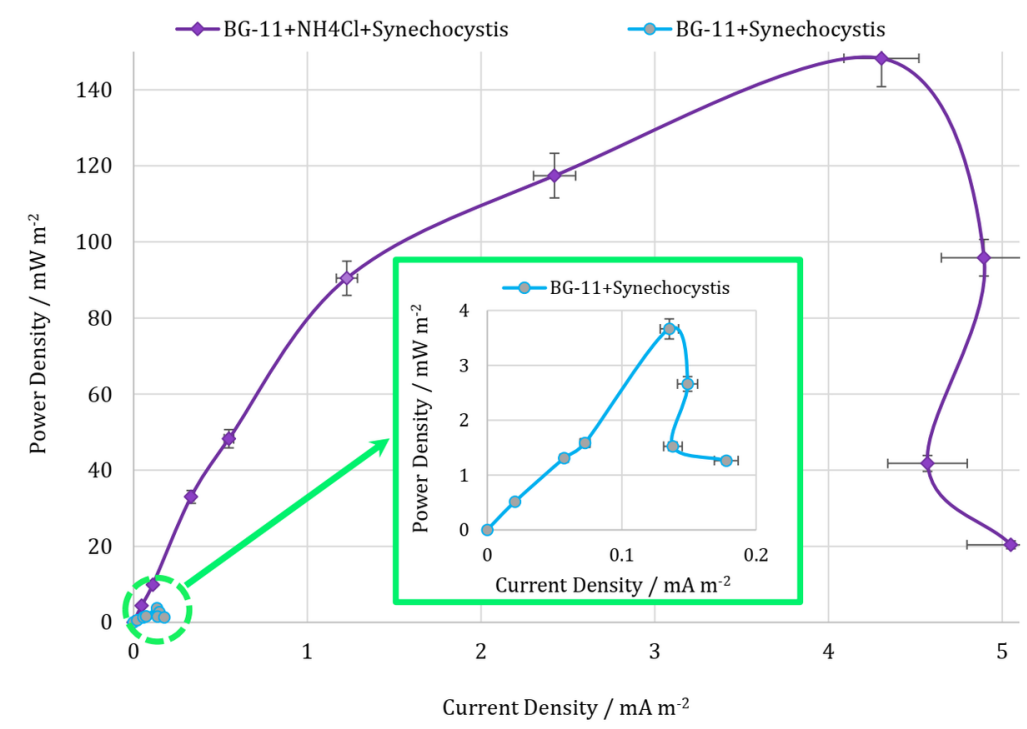


(D)

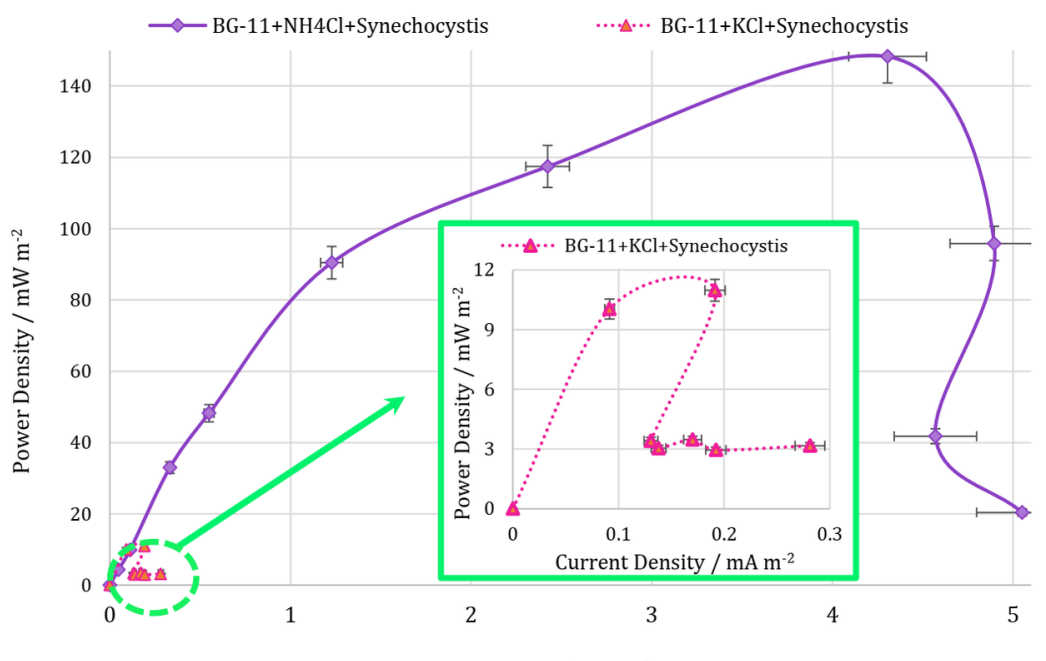

(E)

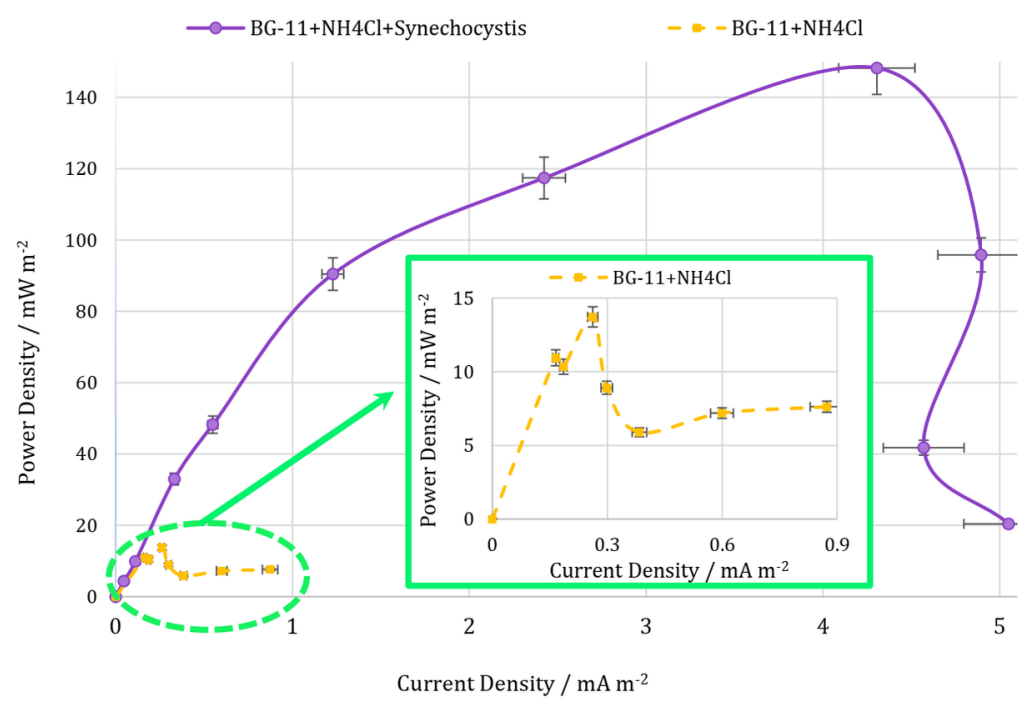

Fig. 5. (A) The current evolution, (B) polarization and (C), (D), and (E) power density curves for different culture media. The error bars represent the variation of obtained results among repeated experiments. 


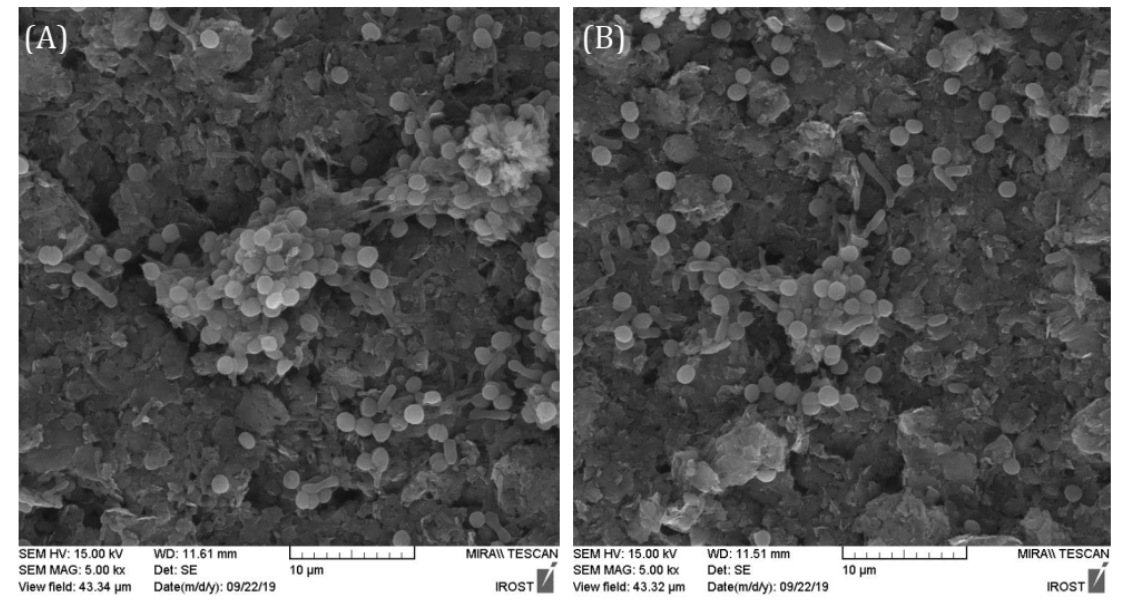

Fig. 6. Field emission scanning electron microscopy (FESEM) of Synechocystis biofilm in (A) BG-11, and (B) $\mathrm{BG}-11+\mathrm{NH}_{4} \mathrm{Cl}$ on the anode surface at 5000 magnification. 